\title{
Correlation between the time elapsed after liposuction and the risk of fat embolism: An animal model
}

\author{
Kwang-Ryeol Lim ${ }^{1}$, Jeong-Min Cho ${ }^{1}$, Chung-Min Yoon ${ }^{1}$, Keun-Cheol Lee ${ }^{1}$, Sang-Yun Lee ${ }^{2}$, \\ Mi-Ha Ju ${ }^{3}$ \\ ${ }^{1}$ Department of Plastic and Reconstructive Surgery, Dong-A University School of Medicine, Busan; ${ }^{2}$ Ellium Women's Hospital, Busan; \\ ${ }^{3}$ Department of Pathology, Dong-A University School of Medicine, Busan, Korea
}

Background Liposuction has become one of the most frequently performed procedures in the field of aesthetic surgery. Fat embolism syndrome after liposuction can easily be overlooked or underestimated; however, occasionally, fulminating fat embolism syndrome can develop and lead to a critical situation within 2-3 days after lipoplasty. Changes over time in the amount of circulating fat particles and the histology of major organs have not yet been studied.

Methods This study was conducted using 18 male Sprague-Dawley rats aged 12 weeks and weighing 500-628 $\mathrm{g}$ (average, $562 \mathrm{~g}$ ). Fifteen rats were used as the experimental group and 3 as the control group. Under general anesthesia, tumescent-technique liposuction was performed at the lateral flank areas and abdomen for 1 hour. Blood, lung, and brain tissue specimens were obtained at 1 hour, 1 day, and 2 days after the liposuction procedure.

Results The average number of fat particles in the blood samples was 25,960/dL at 1 hour, $111,100 / \mathrm{dL}$ at 24 hours, and $21,780 / \mathrm{dL}$ at 48 hours. The differences between study groups were statistically significant. Both intravascular and extravascular fat particles with inflammation were seen in all 15 rats, as were inflammatory cell infiltration, hemorrhage, and consolidation with shrinkage of the lung alveoli.

Conclusions These results imply that there is a strong possibility of fat embolism syndrome after liposuction in real clinical practice, and the first 24-48 hours after the operation were found to be the most important period for preventing pulmonary embolism and progression to fulminating fat embolism syndrome.

Keywords Liposuction / Adipose tissue / Embolism / Fat embolism syndrome
Correspondence: Kwang-Ryeol Lim Department of Plastic and Reconstructive Surgery, Dong-A University School of Medicine, 26 Daesingongwon-ro, Seo-gu, Busan 49201, Korea Tel: +82-51-240-5410 Fax: +82-51-248-1527 E-mail: Iky0707ps@hanmail.net

This study was supported by research funds from Dong-A University.

Received: 17 Aug $2017 \bullet$ Revised: 12 Oct $2017 \bullet$ Accepted: 18 Oct 2017

pISSN: 2234-6163 • elSSN: 2234-6171 • https://doi.org/10.5999/aps.2017.01347 • Arch Plast Surg 2018;45:14-22

\section{INTRODUCTION}

Fat embolism syndrome is uncommon after liposuction, although it is a well-known complication of long bone fractures, cardiopulmonary resuscitation, and lipid infusion for parenteral feeding [1]. Since the introduction of lipoplasty in the mid1970s [2], liposuction has become one of the most popular cosmetic surgical procedures because of an increased interest in 
body shape [3]. Therefore, the incidence of fat embolism syndrome after liposuction has increased.

The severity of fat embolism syndrome varies widely, from mild respiratory distress in the subclinical state to profound refractory hypoxemia leading to coma and death [4]. The 3 main symptoms of fat embolism syndrome-acute respiratory distress, neurologic dysfunction, and dermatological lesions-appear 12-72 hours after the precipitating event. However, since one or more of these findings may be absent, it is difficult to make a clinical diagnosis after a surgical procedure such as liposuction.

During liposuction, fat tissue in the subcutaneous layer is injured and destroyed by a suction cannula and negative pressure. Most of the destroyed tissue fragments are removed, but some of them drain into the systemic circulation through the traumatized vessels [5]. Fat particles that enter the venous circulation via ruptured vessels can become emboli in major organs such as the lung, brain, kidney, and skin. These emboli interfere with the bloodstream, eventually obstructing blood flow and gas exchange in the lung [6]. Additionally, bioactive toxic substances, such as free fatty acid chylomicrons originating from the destroyed adipocytes $[7,8]$ and hormones released after trauma, enhance inflammation and cell destruction in fat embolism syndrome [9].

The clinical pattern of fat embolism syndrome is usually nonspecific. Accordingly, the incidence of fat embolism syndrome after liposuction may be underestimated [10]. Even though most cases of fat embolism syndrome are subclinical or have minor symptoms initially, surgeons must keep in mind that they may progress to fulminating fat embolism syndrome, which can be fatal. Inappropriate procedures that result in massive tissue destruction (especially in cases of large-volume liposuction) and overlooked minor fat embolism syndrome in patients with underlying chronic disease can lead to fulminating fat embolism syndrome, which results in a critical situation within 2-3 days of liposuction [11]. Therefore, this study was designed to elucidate the serial changes in the amount of fat emboli in the blood and histological findings of the lung and brain during the first 48 hours after liposuction.

\section{METHODS}

\section{Clinical subjects}

Sprague-Dawley rats were used as an animal model with similar physiology to human beings. This study was performed using 18 rats aged 12 weeks weighing 500-628 g (average, 562 g). Fifteen rats were used as the study group, and 3 rats were used as the control group (Table 1). Blood, lung, and brain tissue speci-

\section{Table 1. Weight distribution of the studied rats}

\begin{tabular}{|lcc|}
\hline Group $(\mathbf{n = 1 8 )}$ & Range $(\mathbf{g})$ & Mean \pm SD $(\mathbf{g})$ \\
\hline Control $(n=3)$ & 530 to 580 & $560 \pm 21.60$ \\
$1 \mathrm{hr}(n=5)$ & 530 to 601 & $557 \pm 25.53$ \\
$24 \mathrm{hr}(n=5)$ & 500 to 628 & $587 \pm 43.26$ \\
$48 \mathrm{hr}(n=5)$ & 485 to 582 & $541 \pm 36.83$ \\
\hline SD, standard deviation. & & \\
\hline
\end{tabular}

mens were obtained without liposuction in the control group. Each of the 3 study groups consisted of 5 rats that underwent liposuction. In group 1, blood, lung, and brain tissue specimens were obtained 1 hour after liposuction, while in groups 2 and 3 , the specimens were obtained 24 hours and 48 hours after liposuction, respectively. The experimental protocol, including the use of animals in the study, was approved by the Institutional Animal Care and Use Committee, Dong-A University, Korea (DIACUC-Approval-16-21).

\section{Procedure}

Liposuction was performed using the syringe method as in clinical settings [5]. Experiments were carried out under general anesthesia. After inductive inhalation anesthesia using ether, maintenance was achieved by intraperitoneal injections of ketamine Hydrogen chloride ( $\mathrm{HCl}, 40 \mathrm{mg} / \mathrm{kg}$ ).

The procedure was conducted using medical-grade stainless steel liposuction cannulas in 2 sizes. The cannula had an inner diameter of 2 or $3 \mathrm{~mm}$ and a length of $20 \mathrm{~cm}$ with a rounded bullet tip, smooth surface, and 3 openings on the side through which the aspirated fat drained into the syringe.

Instead of a suction aspirator, a $10-\mathrm{mL}$ syringe connected to the cannula was used. The connection between the syringe and cannula was designed to be tight enough for air not to leak, while still enabling it to be easily plugged and unplugged. During the procedure, the suction pressure was kept at about -50 $\mathrm{kPa}$ by pulling the piston $3 \mathrm{~mL}$ backward with $3 \mathrm{~mL}$ of air in the syringe. As the suction aspirate flowed into the syringe, the negative pressure of the syringe decreased. To maintain the $-50 \mathrm{kPa}$ pressure consistently, the piston was pulled backward manually, keeping pace with the volume of aspirate within the syringe.

After anesthesia, each rat was placed on the operating table in supine position in a manner similar to that used in clinical practice, and the abdomen and flank areas were shaved.

As a tumescent solution, $50-60 \mathrm{~mL}$ of normal saline (approximately $10 \%$ of the animal's weight) was injected into the fat deposits in the flank and abdomen areas of the rat. Liposuction was started approximately 30 minutes after injection of the tumescent solution. During liposuction, minimal incision and dissection were performed on both sides of the groin to facilitate 
fat aspiration in the subcutaneous layer and to avoid trauma to the surrounding tissues. Liposuction was then performed in the usual clinical manner described above for 30 minutes on each side, for a total of 1 hour.

During the procedure, care was taken to keep the cannula within the subcutaneous fat deposit layer to prevent injury to the surrounding tissues, including the muscle. To accomplish this, the cannula was positioned just beneath the skin layer. The aspirate collected in the syringe was then transferred into $50-\mathrm{mL}$ Falcon tubes after the procedure.

\section{Collection and examination}

Blood samples were collected from both study and control animals in the same way. Namely, $2 \mathrm{~mL}$ of blood was collected from all animals after liposuction, just before sacrifice. Cardiac puncture was performed, and blood was drained through a catheter inserted into the left side of the heart and then held on ice until staining with Oil-Red $\mathrm{O}$ to identify the fat molecules. The blood samples were transferred into appropriately labeled Falcon tubes, and then $0.2 \mathrm{~mL}$ of Oil-Red $\mathrm{O}$ was added to each sample using a pipette. The contents of each tube were then mixed together using a whirl mixer for 5 minutes, and samples were placed on a miniorbital shaker (at a speed of $75 \mathrm{rpm}$ ) for 15 minutes. Finally, the samples were centrifuged for 17 minutes at $-4^{\circ} \mathrm{C}$ and 5,000 rpm. The supernatant was then collected using a Liquipette (Elkay, Basingstoke Hampshire, United Kingdom) and placed on a hemocytometer slide (Neubauer chamber, Celeromics, Grenoble, France). These slides were then examined under a light microscope for the presence of stained fat particles to confirm systemic fat mobilization following liposuction. The fat particles were counted manually and photographed.

Histological specimens were obtained simultaneously from the lungs and brains of the rats. Half of each organ was frozen at $-20^{\circ} \mathrm{C}$ in a cryotome for identification of the fat particles by Sudan Black-B staining. Sudan Black-B is the most sensitive and versatile lysochrome (fat-soluble) dye used to stain lipids; with this dye, fat appears blue-black $[12,13]$. The other half of the organs was kept in $10 \%$ neutral phosphate-buffered formalin until hematoxylin and eosin staining for the histological examination.

The stained organs were wax-impregnated and sectioned using a cryotome, and sections were then examined under the microscope for the presence of lipid deposits as evidence of fat embolism after liposuction.

\section{Statistical analysis}

Experimental values are presented as the mean \pm standard deviation. Statistical significance was determined by the Kruskal-
Wallis test using SPSS ver. 18 (SPSS Inc., Chicago, IL, USA). Pvalues $<0.05$ were considered statistically significant, according to the Fisher least significant difference analysis.

\section{RESUITS}

\section{Aspirated material}

The suction aspirate primarily consisted of fat tissue, blood, serous fluid, and the injected tumescent solution, and its amount ranged from 7.5 to $12.5 \mathrm{~mL}$ (average, $10.2 \mathrm{~mL}$ ) in the 1-hour group, from 10.0 to $17.5 \mathrm{~mL}$ (average, $14.3 \mathrm{~mL}$ ) in the 24-hour group, and from 9.0 to $11.0 \mathrm{~mL}$ (average, $10.0 \mathrm{~mL}$ ) in the 48 hour group.

The amount of aspirated fat tissue ranged from 3.5 to $5.0 \mathrm{~mL}$ (average, $4.2 \mathrm{~mL}$ ) in the 1-hour group, from 4.0 to $8.0 \mathrm{~mL}$ (average, $5.5 \mathrm{~mL}$ ) in the 24-hour group, and from 5.0 to $5.5 \mathrm{~mL}$ (average, $5.1 \mathrm{~mL}$ ) in the 48-hour group (Table 2). Although the proportion of fat tissue within the suction aspirate was different among the 3 study groups, this difference was not statistically significant $(\mathrm{P}>0.05)$.

\section{Examination of blood samples}

The amount of fat particles detected in blood was calculated. Nothing of significance was seen in the blood samples of the control group, but many Oil-red $\mathrm{O}$ stained fat particles of various sizes and morphological features were observed in all the rats in the 3 study groups. Typical specimen slides are shown in Fig. 1.

Table 3 shows the distribution of the number of fat particles over time after liposuction. In the 1-hour samples, the number of particles ranged from $14,300 / \mathrm{dL}$ to $51,700 / \mathrm{dL}(25,960 \pm$ $14,636 / \mathrm{dL})$, while in the 24-hour samples, the number ranged from $96,800 / \mathrm{dL}$ to $134,200 / \mathrm{dL}(111,100 \pm 13,015 / \mathrm{dL})$, and in the 48-hour samples from $7,700 / \mathrm{dL}$ to $34,100 / \mathrm{dL}(21,780 \pm$ $9,971 / \mathrm{dL})$. In the control group, nothing of significance was seen in any of the blood samples.

The number of fat particles in the control and 3 study groups,

Table 2. Amount of suction aspirate and fat tissue within the aspirate

\begin{tabular}{|c|c|c|c|c|}
\hline \multirow{2}{*}{ Group $(n=18)$} & \multicolumn{2}{|c|}{ Suction aspirate $(\mathrm{mL})$} & \multicolumn{2}{|c|}{ Fat tissue $(\mathrm{mL})$} \\
\hline & Range & Mean \pm SD & Range & Mean \pm SD \\
\hline Control $(n=3)$ & 0 & 0 & 0 & 0 \\
\hline $1 \mathrm{hr}(n=5)$ & 7.5 to 12.5 & $10.2 \pm 0.57$ & 3.5 to 5.0 & $4.2 \pm 0.51$ \\
\hline $24 \mathrm{hr}(\mathrm{n}=5)$ & 10.0 to 17.5 & $14.3 \pm 1.44$ & 4.0 to 8.0 & $5.5 \pm 1.38$ \\
\hline $48 \mathrm{hr}(\mathrm{n}=5)$ & 9.0 to 11.5 & $10.0 \pm 0.25$ & 5.0 to 5.5 & $5.1 \pm 0.20$ \\
\hline
\end{tabular}




\section{Fig. 1. Oil-Red 0-stained fat particles in the blood}

Nothing of significance was seen in the control group (A), but many fat particles were seen in all study groups (B-D). The highest fat particle count was seen in the 24-hour group (C). (A) Control group. (B) 1 hour. (C) 24 hours. (D) 48 hours. (A-D) $\times 200$.
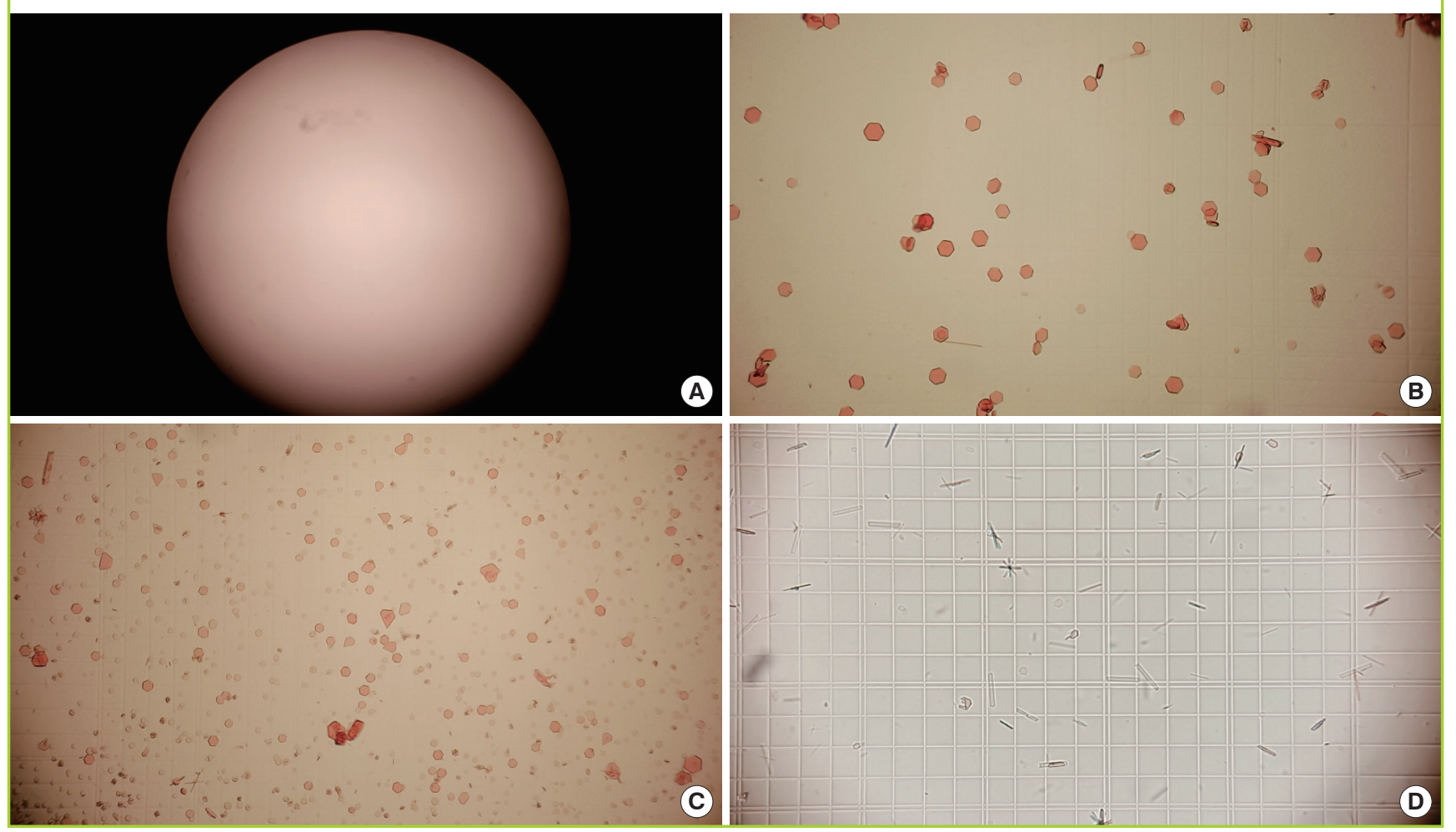

Table 3. Fat particle counts in the blood samples in the 3 study groups

\begin{tabular}{|c|c|c|c|c|}
\hline \multirow{2}{*}{$\begin{array}{l}\text { Study } \\
\text { group } \\
(n=15)\end{array}$} & \multicolumn{2}{|c|}{ Range (/dL) } & \multicolumn{2}{|c|}{$\begin{array}{c}\text { Mean } \pm \text { standard } \\
\text { deviation }(/ \mathrm{dL})\end{array}$} \\
\hline & Group & Control & Group & Control \\
\hline $1 \mathrm{hr}(\mathrm{n}=5)$ & 14,300 to 51,700 & 0 & $25,960 \pm 14,636$ & 0 \\
\hline $24 \mathrm{hr}(\mathrm{n}=5)$ & 96,800 to 134,200 & 0 & $111,100 \pm 13,015$ & 0 \\
\hline $48 \mathrm{hr}(\mathrm{n}=5)$ & 7,700 to 34,100 & 0 & $21,780 \pm 9,971$ & 0 \\
\hline
\end{tabular}

and the intergroup differences among the 3 study groups, were compared in SPSS. The difference between the study groups as a whole and the control group was statistically significant $(\mathrm{P}<$ 0.001). The intergroup differences between the study groups were also statistically significant (between 1 hour and 24 hours, $\mathrm{P}<0.05$; between 24 hours and 48 hours, $\mathrm{P}<0.05$ ) (Fig. 2).

\section{Histological examinations}

Lung and brain tissues obtained from the control and study groups were evaluated. The tissue specimens were categorized depending on the presence of intravascular and extravascular fat drops and the degree of inflammation, and then the results were statistically analyzed.

\section{Fig. 2. Mean fat particle counts}

Statistically significant differences were found between the 1-hour and 24-hour groups and between the 24-hour and 48-hour groups $(P<0.05)$.

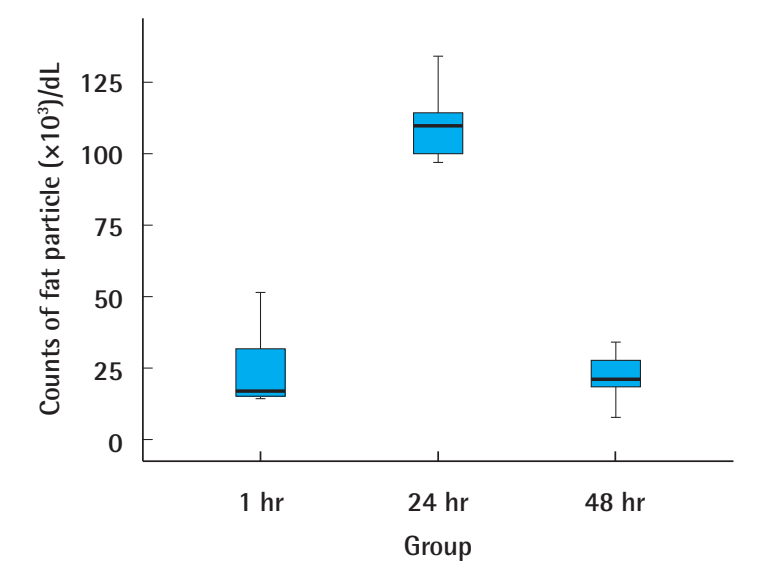

\section{Lung specimens}

Lipid deposits were observed in the lungs of the animals in all 3 study groups, but not in the control group (Fig. 3). In general, both intravascular and extravascular fat deposits were identified in all 15 rats in the 3 study groups. More spillage of fat particles 


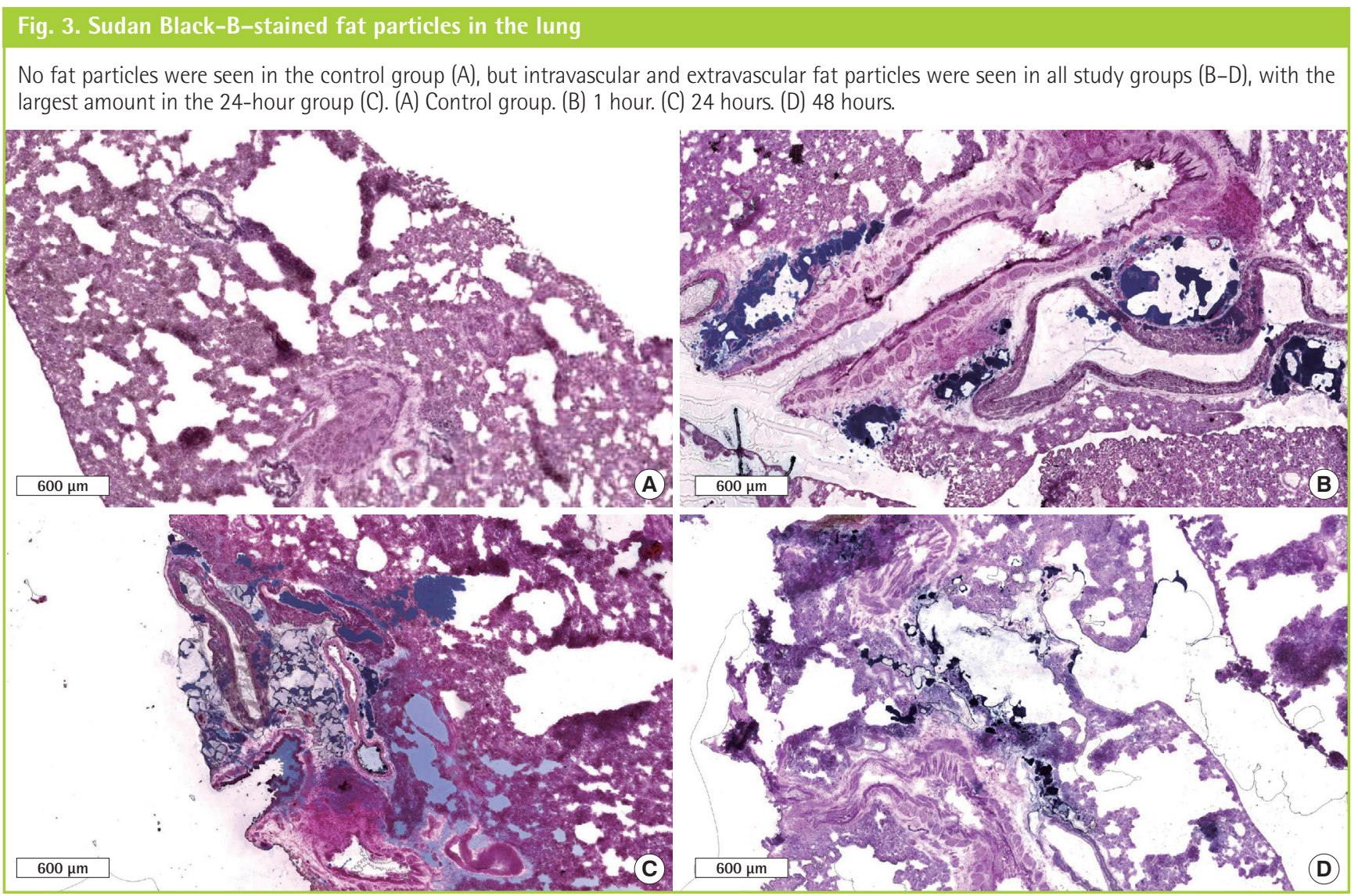

\begin{tabular}{|c|c|c|c|c|}
\hline \multirow{2}{*}{ Group $(n=18)$} & \multicolumn{4}{|c|}{ Intravascular/Extravascular (no. of slides) } \\
\hline & Negative & Mild & Moderate & Severe \\
\hline Control $(n=3)$ & $3 / 3$ & $0 / 0$ & $0 / 0$ & $0 / 0$ \\
\hline $1 \mathrm{hr}(\mathrm{n}=5)$ & $0 / 0$ & $5 / 5$ & $0 / 0$ & $0 / 0$ \\
\hline $24 \mathrm{hr}(\mathrm{n}=5)$ & $0 / 0$ & $1 / 2$ & $2 / 3$ & $2 / 0$ \\
\hline $48 \mathrm{hr}(\mathrm{n}=5)$ & $0 / 0$ & $0 / 0$ & $5 / 0$ & $0 / 5$ \\
\hline
\end{tabular}

into the extravascular space was observed over time (Table 4). The difference between the study and control animals was significant $(\mathrm{P}<0.001)$.

Inflammation was evaluated according to the degree of hemorrhagic congestion, consolidation, and inflammatory cell infiltration. All 15 specimens in the 3 study groups showed pathologic inflammation (Fig. 4). Specifically, at 1 hour after liposuction, mild to moderate congestion and consolidation appeared. Over time, inflammation was aggravated, and the 48-hour specimens showed the most severe inflammatory findings of massive cell infiltration, hemorrhage, and shrinkage of the lung alveoli (Table 5).

\begin{tabular}{|c|c|c|c|c|}
\hline \multirow{2}{*}{ Group $(n=18)$} & \multicolumn{4}{|c|}{$\begin{array}{l}\text { Congestion/Consolidation/Bronchial } \\
\text { inflammation (no. of slides) }\end{array}$} \\
\hline & Negative & Mild & Moderate & Severe \\
\hline Control $(n=3)$ & $3 / 3 / 3$ & $0 / 0 / 0$ & $0 / 0 / 0$ & $0 / 0 / 0$ \\
\hline $1 \mathrm{hr}(\mathrm{n}=5)$ & 0/0/0 & $2 / 2 / 3$ & $3 / 3 / 2$ & 0/0/0 \\
\hline $24 \mathrm{hr}(\mathrm{n}=5)$ & 0/0/0 & $0 / 2 / 0$ & $4 / 3 / 5$ & $1 / 0 / 0$ \\
\hline $48 \mathrm{hr}(\mathrm{n}=5)$ & 0/0/0 & $0 / 2 / 0$ & $0 / 3 / 0$ & $5 / 0 / 5$ \\
\hline
\end{tabular}

\section{Brain specimens}

There was no evidence of lipid deposits in the brains of any of the control group animals (Fig. 5A). In 13 of the 15 studied rats, no fat particles were observed upon histological examinations (Table 6). Only a few suspicious, very small fat particles were seen in 2 animals in the 24-hour group (Fig. 5B).

\section{DISCUSSION}

Liposuction is a surgical procedure to remove the excess fat tissue from overweight individuals that is popular in many countries and usually has few side effects. The postoperative compli- 


\section{Fig. 4. Hematoxylin and eosin-stained lung specimens}

Nothing of significance was seen in the control group (A), but many inflammatory findings such as inflammatory cell infiltration, consolidation, and hemorrhagic congestion were noted in the study groups (B-D). Black arrows indicate inflammatory cells, hemorrhagic congestion, and consolidation. (A) Control group. (B) 1 hour. (C) 24 hours. (D) 48 hours.
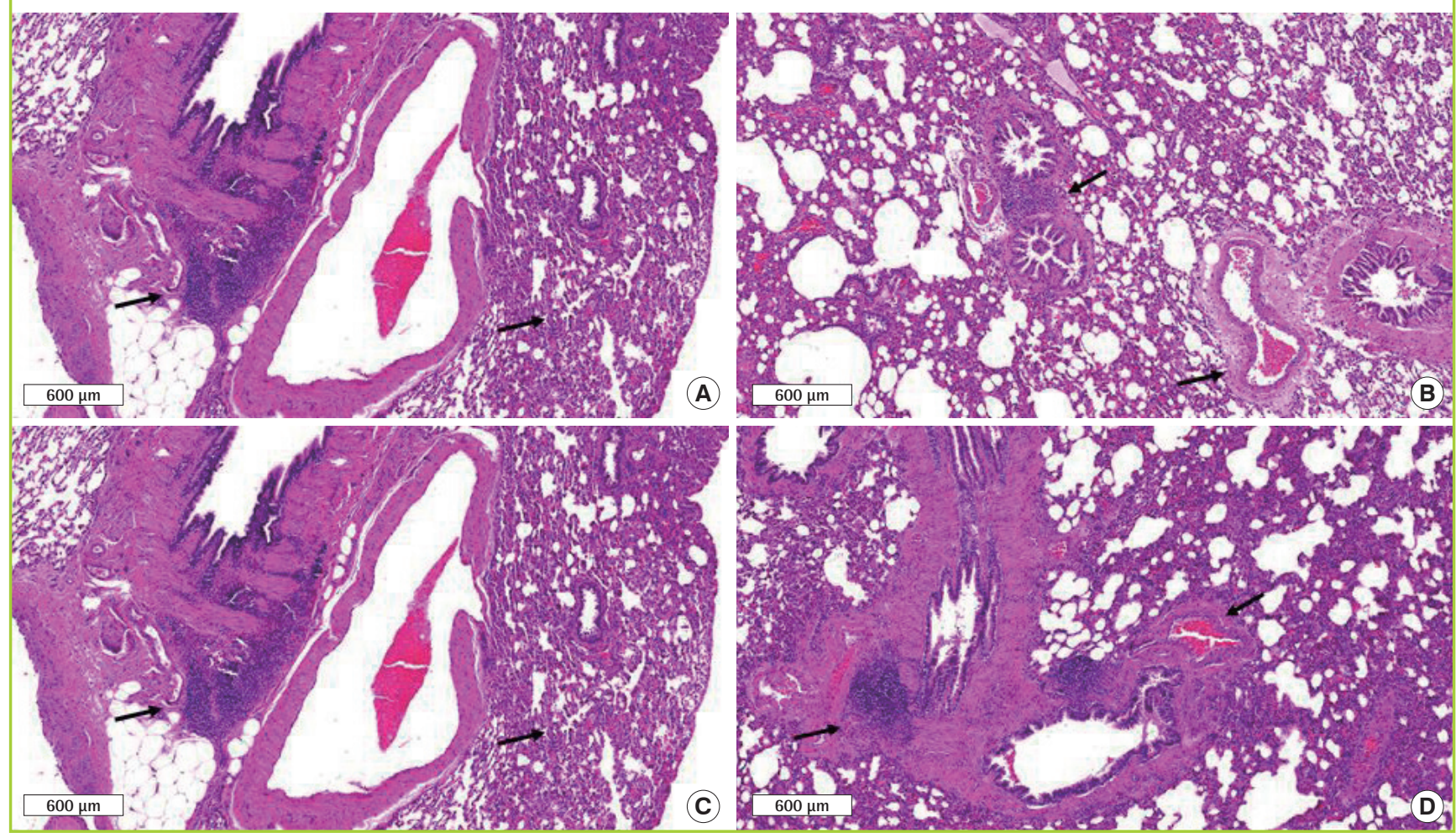

\section{Fig. 5. Sudan Black-B-stained brain specimen}

(A) Nothing of significance was seen in the control group. (B) A few tiny, suspicious deposits (potential fat emboli) can be seen in brain samples from the 24-hour study group.
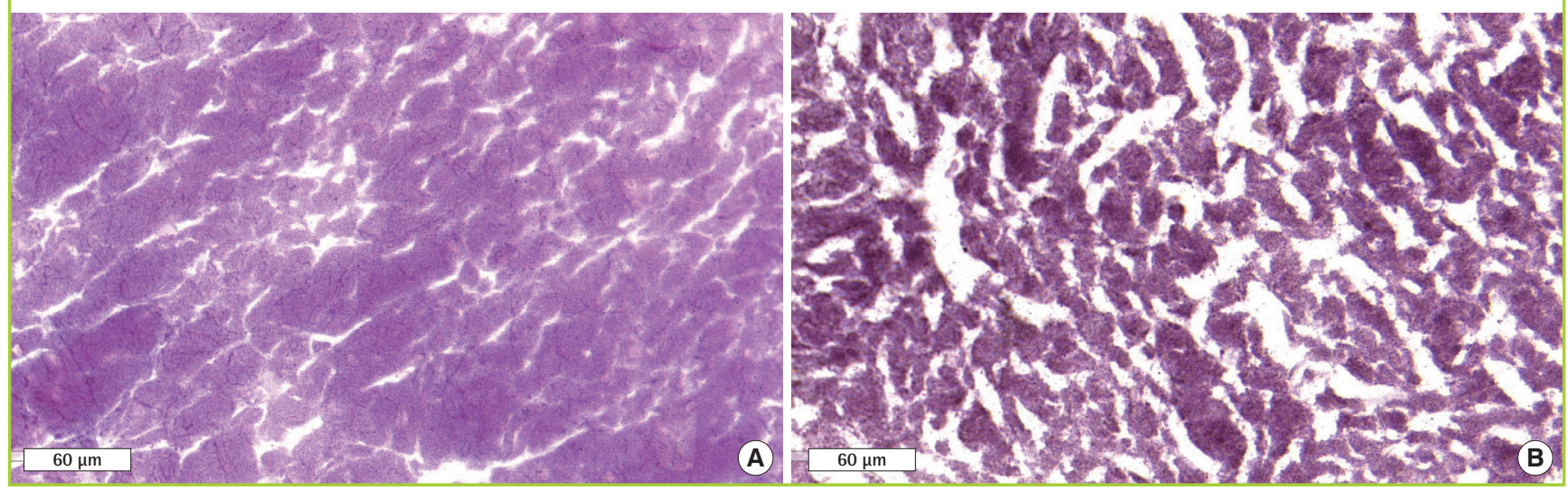

cation rate of liposuction has been reported to be $5 \%-10 \%$, but most complications were minor, such as pain, edema, hematoma, mild dyspnea, and headache [1]. The primary self-limiting complication of liposuction is fat embolism syndrome. Although the incidence of fat embolism syndrome after liposuction is very low, it can be fatal.

Fat embolism syndrome was first described by Gurd [14] in

\begin{tabular}{|lc|}
\hline Table 6. Histologic results of the brain specimens \\
\hline Group & Lipid deposits \\
\hline Control & Not seen \\
$1 \mathrm{hr}$ & Not seen \\
$24 \mathrm{hr}$ & Suspicious deposits in 2 rats \\
$48 \mathrm{hr}$ & Not seen \\
\hline
\end{tabular}


1970. The 3 classic symptoms of fat embolism syndrome-respiratory distress, cerebral dysfunction, and petechial rashusually appear within $24-72$ hours after liposuction in $85 \%$ of the affected patients [15], with a symptom-free interval of 1230 hours after injury [16]. Major fat embolism syndrome has been suggested to cause clinical symptoms such as tachycardia, tachypnea, fever, and hypoxemia. Arterial blood gas analysis, as well as blood, urine, and sputum testing, could be helpful for the diagnosis of fat embolism syndrome. Chest radiography, noncontrast computed tomography (CT) scans, high-resolution $\mathrm{CT}$, ventilation-perfusion imaging, and magnetic resonance imaging can also be used to diagnose this condition. In chest Xrays, signs of generalized pulmonary interstitial and alveolar opacification appeared within the first 3 days after liposuction in affected patients [1].

Fat embolism syndrome has 2 pathophysiological processes. First, the fat particles produced by the destruction of adipocytes during cannula movement in the subcutaneous layer enter the surrounding ruptured venules. These fat particles become emboli in the bloodstream and are transported to the pulmonary vascular beds [15]. Fat emboli ranging in size from $10 \mu \mathrm{m}$ to 40 $\mu \mathrm{m}$ in diameter have been found to block pulmonary blood vessels, leading to increased pulmonary arterial pressure and hypoxia $[3,4]$. This increased pressure could push the fat emboli into the general systemic circulation, resulting in major organ embolism [17]. Second, free fatty acids, such as chylomicrons, are generated from the broken particles of fat globules, and then act as toxins toward pneumocytes and the capillary endothelium $[5,6]$.

Fulminant fat embolism syndrome is a severe manifestation of acute cardiovascular pulmonary obstruction caused by the sudden intravascular release of a large amount of fat emboli. Severe pulmonary hypertension, right heart failure, shock, and even death often occur within the first 48 hours of injury in affected individuals [18].

In summary, fat embolism syndrome is believed to be caused by vascular obstructions arising from emboli and the toxic effects of bioactive substances such as free fatty acids. The presence of fat particles in the systemic circulation and fat embolism in major organs after liposuction have been previously postulated $[7,8,12,14]$. Unfortunately, changes over time in the quantity of circulating fat particles and the histology of major organs after liposuction have yet to be investigated.

In this study, a suction system was used with a rat model to explore these questions. Although the amount of aspirated fat in the 3 study groups differed, the differences were not statistically significant $(\mathrm{P}>0.05)$. There were no problems associated with the examination and analysis of blood samples or the histopath- ological analysis of the lungs and brain.

El-Ali and Gourlay [19] reported 38,427 and 89,254 fat particles per deciliter in blood samples obtained 30 minutes and 60 minutes after liposuction, respectively. In our results, fat particles were consistently present throughout the experimental period, with quantities of $25,960 / \mathrm{dL}, 111,100 / \mathrm{dL}$, and 21,780/ $\mathrm{dL}$ in blood samples obtained 1 hour, 24 hours, and 48 hours after liposuction, respectively. The difference in the number of fat particles between the 3 groups and the control samples was statistically significant $(\mathrm{P}<0.001)$. In addition, statistically significant differences were found between the samples from the 1-hour group and the 24-hour group $(\mathrm{P}<0.05)$, and between the samples from the 24-hour group and the 48-hour group $(\mathrm{P}<0.05)$.

In this study, the levels of fat particles in the 24-hour group were nearly 4 times higher than those of the 1 -hour or 48 -hour groups. These findings demonstrate that fat mobilization during first 24 hours after liposuction was a cumulative process, and that large amounts of fat particles might remain within and around the operative field for 24 hours after liposuction. During the first 24 hours, these hidden fat particles continuously entered the ruptured vessel. Fat mobilization decreased after 24 hours, which is why the fat cell counts in the 24-hour group were much higher than those of the 48-hour group.

The risk of fat mobilization and fat embolism were highest around 24 hours after liposuction. Therefore, the first symptoms of fat embolism syndrome usually appeared at this time.

The histological examinations showed no abnormalities in the control group. The fat particles released after liposuction traveled first to the heart and then to the lungs, which were the first organ to entrap the fat particles. Indeed, Sudan black staining revealed fat particles in the lungs of all 15 rats in the 3 study groups in both the intravascular and extravascular spaces, but not in the control group. It was found that macrophages contained fat particles. In the 1-hour group, few fat particles were seen in the intravascular or extravascular spaces. Over time, fat particles spilled out into the extravascular interstitial space, lung parenchymal tissue, and bronchioles. As a result, more extravascular spillage was noted in the 24- and 48-hour groups (Table 4).

In other studies of liposuction using a porcine lung model, patchy petechial hemorrhages were observed on the pleural surface, as well as marked hemorrhagic congestion and evidence of pulmonary edema [13]. In this study, indicators of inflammation such as edema, hemorrhagic congestion, consolidation with shrinkage of alveoli, and inflammatory cell infiltration causing pulmonary edema were found. The inflammatory findings of each group were categorized according to severity (Table 5). Minimal to moderate inflammation was seen in the early pe- 
riod after liposuction, but marked cell infiltration and hemorrhagic congestion were seen over time. These findings underscore the implications of the fat particle counts in blood samples, in that they indicate that fat embolism syndrome appeared around 24 hours after liposuction and peaked at about 48 hours after liposuction. However, further studies are needed to better understand the systemic mobilization of fat particles and organ embolism after liposuction. El-Ali and Gourlay [19] reported that fat particles were found in the lungs of $100 \%$ of experimental animals, and in the brains of $10 \%$. It is possible that the lungs filtered fat particles from the circulatory system, resulting in fewer emboli in other organs.

In exceptional cases, tiny deposits might escape the lung filter and end up in the brain or other organs. In this study, suspected fat particles were found in 2 brain specimens from the 24-hour group, but because they were so small, they could not be confirmed as fat particles (Table 6). The control group had normal histological features without fat particles in the brain specimens.

This study characterized the presence of fat emboli in the blood and lungs, and demonstrated inflammatory findings in the lungs after liposuction. In other words, there is always a risk of fat mobilization into the lungs and brain via the systemic circulation, resulting in major organ fat embolism during the early and late postoperative period.

As discussed above, the clinical picture of fat embolism syndrome ranges from mild subclinical hypoxia to severe respiratory distress, and even coma and death. It is important to select patients and liposuction techniques with the goal of preventing fat embolism syndrome. Closer observation and careful evaluation of the early symptoms after operation are useful for making an early diagnosis. If fat embolism syndrome is suspected, appropriate aggressive therapy should be conducted to reduce morbidity and to prevent progression to fulminating fatal fat embolism syndrome.

This study was a first step toward elucidation of the post-liposuction time corresponding to systemic fat mobilization and major organ embolism. However, this study has the limitation that actual clinical conditions were not reproduced completely in this study, in that the ordinary tumescent solution (made up of lidocaine and epinephrine with or without Bivon) that is used in actual clinical practice was not employed. Thus, additional experiments under actual clinical conditions need to be conducted to obtain more meaningful results in follow-up research. In addition, further large-scale studies with subdivided study groups are needed to obtain a better understanding of fulminating fatal fat embolism syndrome following liposuction.

\section{CONFLICT OF INTEREST}

No potential conflict of interest relevant to this article was reported.

\section{REFERENCES}

1. Wang HD, Zheng JH, Deng CL, et al. Fat embolism syndromes following liposuction. Aesthetic Plast Surg 2008;32: 731-6.

2. Coleman WP 3rd, Hanke CW, Orentreich N, et al. A history of dermatologic surgery in the United States. Dermatol Surg 2000;26:5-11.

3. Rohrich RJ. The increasing popularity of cosmetic surgery procedures: a look at statistics in plastic surgery. Plast Reconstr Surg 2000;106:1363-5.

4. Glover P, Worthley LI. Fat embolism. Crit Care Resusc 1999;1:276-84.

5. Levy D. The fat embolism syndrome: a review. Clin Orthop Relat Res 1990;(261):281-6.

6. Iverson RE, Lynch DJ. Practice advisory on liposuction. Plast Reconstr Surg 2004;113:1478-90.

7. Liljedahl SO, Westermark L. Aetiology and treatment of fat embolism: report of five cases. Acta Anaesthesiol Scand 1967;11:177-94.

8. Ross RM, Johnson GW. Fat embolism after liposuction. Chest 1988;93:1294-5.

9. Illouz YG. Complications of liposuction. Clin Plast Surg 2006;33:129-63.

10. Fourme T, Vieillard-Baron A, Loubieres Y, et al. Early fat embolism after liposuction. Anesthesiology 1998;89:782-4.

11. Christman KD. Death following suction lipectomy and abdominoplasty. Plast Reconstr Surg 1986;78:428.

12. Kiernan JA. Histological and histochemical methods: theory and practice. Oxford: Pergamon Press; 1981.

13. Kenkel JM, Brown SA, Love EJ, et al. Hemodynamics, electrolytes, and organ histology of larger-volume liposuction in a porcine model. Plast Reconstr Surg 2004;113:1391-9.

14. Gurd AR. Fat embolism: an aid to diagnosis. J Bone Joint Surg Br 1970;52:732-7.

15. Shier MR, Wilson RF. Fat embolism syndrome: traumatic coagulopathy with respiratory distress. Surg Annu 1980;12: $139-68$.

16. Gossling HR, Donohue TA. The fat embolism syndrome. JAMA 1979;241:2740-2.

17. Lequire VS, Shapiro JL, Lequire CB, et al. A study of the pathogenesis of fat embolism based on human necropsy material and animal experiments. Am J Pathol 1959;35:999- 
1015.

18. Pell AC, Hughes D, Keating J, et al. Brief report: fulminating fat embolism syndrome caused by paradoxical embolism through a patent foramen ovale. N Engl J Med 1993;329:
926-9.

19. El-Ali KM, Gourlay T. Assessment of the risk of systemic fat mobilization and fat embolism as a consequence of liposuction: ex vivo study. Plast Reconstr Surg 2006;117:2269-76. 